\title{
Parâmetros dinâmicos de movimentos selecionados da Capoeira
}

\author{
Allan Brennecke \\ Alberto C. Amadio \\ Júlio C. Serrão
}

https://doi.org/10.5628/rpcd.05.02.153

\author{
Universidade de São Paulo \\ Escola de Educação Física e Esporte \\ Laboratório de Biomecânica \\ Brasil
}

\section{RESUMO}

Tendo em vista a escassa produção científica com enfoques biológicos sobre a Capoeira, o objetivo deste estudo foi realizar a análise dinâmica de movimentos selecionados da capoeira no âmbito da Força de Reação do Solo (FRS). A amostra constituiu-se de 10 capoeiristas, os quais realizaram sobre uma plataforma de força 4 diferentes movimentos: "Negativa Fechada", "Martelo", "Armada Pulada" e "Parafuso". A análise das curvas de FRS centrou-se: na Força Vertical Máxima (Fy máx), no Tempo até o Pico de Fy máx (TFy máx), no Gradiente de Crescimento de Fy (GC Fy) e no Impulso gerado por Fy nos primeiros 50ms de execução dos movimentos (I50). Refletindo o comportamento da Fy (max) e do T Fy (max), os menores valores para o GC Fy e para o I50 foram obtidos na "Negativa Fechada" $\left(4,91 \pm 4,69 \mathrm{PC} \cdot \mathrm{s}^{-1} ; 1,53 \pm 0,76 \cdot 10^{-2} \mathrm{~kg} \cdot \mathrm{ms}\right)$ e no "Martelo" $\left(2,61 \pm 1,70 \mathrm{PC} \cdot \mathrm{s}^{-1} ; 0,84 \pm 0,41 \cdot 10^{-2} \mathrm{~kg} \cdot \mathrm{ms}\right)$, enquanto a "Armada Pulada" $\left(115,93 \pm 79,84 \mathrm{PC} \cdot \mathrm{s}^{-1} ; 7,54 \pm 3,04 \cdot 10^{-2}\right.$ $\mathrm{kg} \cdot \mathrm{ms})$ e o "Parafuso" $\left(69,17 \pm 65,68 \mathrm{PC} \cdot \mathrm{s}^{-1} ; 6,38 \pm 2,88 \cdot 10^{-2}\right.$ $\mathrm{kg} \cdot \mathrm{ms})$ apresentaram os maiores valores para estas variáveis. O presente estudo traz evidências de que, pelo menos no âmbito das forças externas, a "Negativa Fechada" e o "Martelo" podem ser inseridos nas fases iniciais do programa de treinamento, ao passo que, o "Parafuso" e a "Armada Pulada", movimentos cujas exigências mecânicas e motoras são maiores, poderiam ser inseridos posteriormente no programa de treinamento. Porém, a interpretação dos resultados exige cautela, uma vez que a sobrecarga mecânica recebida depende essencialmente das condições mecânicas das estruturas osteomioarticulares dos praticantes, bem como do nível de condicionamento físico destes.

Palavras-chave: capoeira, força de reação do solo, biomecânica.

\begin{abstract}
Dynamic parameters of selected movements of "Capoeira"

Considering the limited scientific information concerning a biological focus on "Capoeira", the aim of this study was to conduct a dynamic analysis of selected movements of "Capoeira" in the scope of Ground Reaction Force (GRF). The sample consisted of 10 "capoeiristas". All of them performed the following movements: "Negativa Fechada", "Martelo", "Armada Pulada" and "Parafuso" in a Force Plate. The analysis of the GRF curves was centered in the Maximum Vertical Force (Fy max), in the Time to achieve Fy max Peak (TFy max), in the Vertical Force Growth Rate (GR Fy) and in the Impulse generated for Fy in first $50 \mathrm{~ms}$ of movement execution (I50). Reflecting the behaviour of $F y(\max )$ and TFy (max), the smaller values for GR Fy and I50 were obtained by "Negativa Fechada" $\left(4,91 \pm 4,69 \mathrm{PC} \cdot \mathrm{s}^{-1} ; 1,53 \pm 0,76 \cdot 10^{-}\right.$ $2 \mathrm{kgms})$ and by "Martelo" $\left(2,61 \pm 1,70 \mathrm{PC} \cdot \mathrm{s}^{-1} ; 0,84 \pm 0,41 \cdot 10^{-2}\right.$ kgms), while the "Armada Pulada" (115,93 $\pm 79,84 \mathrm{PC} \cdot \mathrm{s}^{-1}$; $\left.7,54 \pm 3,04 \cdot 10^{-2} \mathrm{~kg} \cdot \mathrm{ms}\right)$ and "Parafuso" $\left(69,17 \pm 65,68 \mathrm{PC} \cdot \mathrm{s}^{-1}\right.$; $6,38 \pm 2,88 \cdot 10^{-2} \mathrm{~kg} \cdot \mathrm{ms}$ ) showed the major values for these variables. The present study brings evidences that, at least in the scope of external forces, the "Negativa Fechada" and "Martelo" could be inserted in the initial phases of training program, whereas the "Parafuso" and "Armada Pulada", movements whose mechanical and motor demands are greater, could be approached later in the same program. However, a cautionary note should be present in these results since the received overload depends not only of the osteomioarticular structures conditions of the practiser, but also of their level of physical conditioning.
\end{abstract}

Key Words: "Capoeira”, ground reaction force, biomechanics. 


\section{INTRODUÇÃO}

A escassez de trabalhos que analisam a Capoeira com enfoque biodinâmico, e em especial com atenção para a sobrecarga sobre o aparelho locomotor que esta prática pode ter, é notória $(3,8)$. Tal condição faz com que o praticante de Capoeira, muitas vezes, não tenha um "respaldo teórico que o leve a uma melhor compreensão do próprio corpo e ao conhecimento das vantagens e desvantagens relacionadas com a própria modalidade que pratica (13, p. 27)". Neste cenário, uma das tarefas da Biomecânica é a caracterização e a otimização das técnicas do movimento humano (1).

Observa-se, pois, que a análise dinâmica dos diversos movimentos da Capoeira também não é suficientemente descrita na literatura especializada. Logo, inicia-se este estudo sem um grande suporte de resultados anteriormente obtidos e discutidos. Por outro lado, a descrição precisa de eventos biológicos é o primeiro passo vital para a compreensão de sua causa (7). De maneira semelhante, uma análise dinâmica de um movimento pode proporcionar informações sobre como o movimento é produzido (6). E essas informações, por sua vez, podem direcionar o condicionamento e o treinamento para um determinado movimento.

Desse modo, a partir dos fenômenos determinantes da sobrecarga mecânica torna-se possível interpretar, dentro do domínio da Biomecânica, variáveis que possam ser controladas, como os aspectos da técnica de execução do movimento que podem interferir na determinação e controle da sobrecarga mecânica. Entre os parâmetros externos de bastante influência na sobrecarga sobre o aparelho locomotor destaca-se a Força de Reação do Solo (FRS) (1).

Pelos argumentos expostos, justifica-se a necessidade de se investigar experimentalmente os movimentos da Capoeira por meio de uma análise dinâmica. É objetivo deste trabalho, portanto, a análise dinâmica de movimentos selecionados da Capoeira no âmbito das forças externas que agem no aparelho locomotor. Espera-se que tais resultados possam contribuir para o aperfeiçoamento da prática, tendo em vista as implicações que os achados podem ter para a melhoria do processo ensino-aprendizagem, para o aperfeiçoamento dos processos de treinamento, bem como para a segurança dos praticantes nas habilidades investigadas.

\section{REVISÃO DA LITERATURA}

Uma vez considerado que a análise dinâmica pode, inicialmente, trazer preciosas informações a respeito das habilidades motoras na Capoeira, resta delimitar quais destas habilidades podem ser analisadas. A literatura específica da Capoeira, entretanto, conta com uma vasta e ambígua classificação de habilidades que variam em nome, formas de execução, variações de um mesmo movimento e, ainda, interpretações diferentes de grupo para grupo. Alguns autores $(4,5,13)$ apresentam, em seus trabalhos, tentativas de organizar esse confuso estado. Apesar das divergências existentes e das precárias descrições acerca dos movimentos, estes trabalhos constituem a única fonte de informação disponível sobre os movimentos utilizados na Capoeira.

Parece, no entanto, que todos os autores concordam com relação à "ginga". Esta tornou-se o movimento fundamental possibilitando o equilíbrio dinâmico para dar cadência ao ritmo dos movimentos do corpo. A partir desse movimento é que todos os outros golpes, acrobacias (ou floreios) e fintas serão executados (2).

Com o advento da Luta Regional Baiana, o ensino por seqüências de movimentos foi implantado por Manoel dos Reis Machado, o Mestre Bimba, decidido a metodizar e aperfeiçoar a Capoeira (13). Na análise detalhada dessas seqüências é possível identificar uma presença constante do movimento "Negativa Fechada". Como ilustrado na Figura 1, este movimento é realizado no solo, no qual o praticante, partindo da ginga, leva uma perna com os joelhos estendidos adiante do corpo, enquanto flexiona os joelhos da perna contra-lateral projetando-se em direção ao solo. A queda é freada com o apoio das duas mãos no solo. A seguir o sujeito tenta "encaixar" o dorso do pé da perna estendida a frente no tornozelo do adversário, e com um rápido movimento auxiliado pelo apoio das mãos no solo volta à posição inicial tentando "puxar" com o pé o adversário.
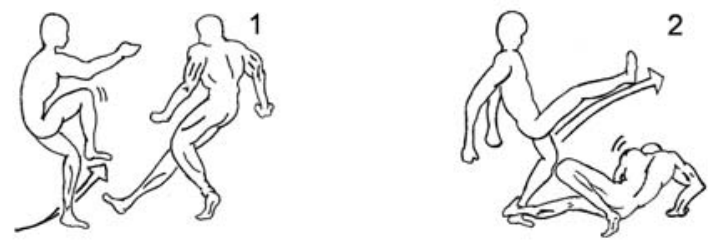

Figura 1 - Fases da Negativa Fechada: (1) "Entrada"; (2) "Encaixe”. 
A constante execução deste movimento nas seqüências de "Bimba" chama a atenção. Ao passo que o praticante deve projetar-se em direção ao solo freando o movimento com as mãos. A sobrecarga recebida por este indivíduo pela Força de Reação do Solo (FRS) é um dado inexistente na literatura. Além da "Negativa Fechada", muitos outros movimentos da Capoeira utilizam-se de aterrissagens cuja sobrecarga no aparelho locomotor é desconhecida. Entre eles pode-se citar a "Armada Pulada" e o "Parafuso". A "Armada Pulada" consiste em, partindo da "ginga", dar um passo à frente, rotacionar o corpo inteiro (cabeça, tronco, pernas e pés) e saltar desprendendo o golpe na fase aérea. A aterrissagem é feita sobre apenas um dos membros inferiores, em particular o mesmo que executou a propulsão do salto (Figura 2).
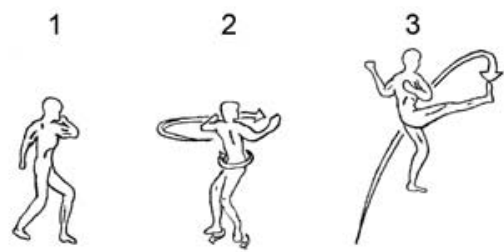

4

Figura 2 - Fases da Armada Pulada: (1) Preparação; (2) Rotação de todo corpo; (3) Salto e chute; (4) Aterrissagem.

O "Parafuso" é semelhante à "Armada Pulada". As diferenças são basicamente duas. A primeira encontra-se durante a fase aérea, na qual após desprender a "Armada Pulada", o praticante ainda executa um chute no mesmo sentido com a outra perna. A segunda diferença está na aterrissagem, pois, devido ao chute que foi adicionado neste golpe, esta é feita não mais com o membro inferior responsável pela propulsão, mas sim, com o contra-lateral. Esta técnica de executar o "Parafuso" é denominada técnica tradicional (8) e está representada na Figura 3
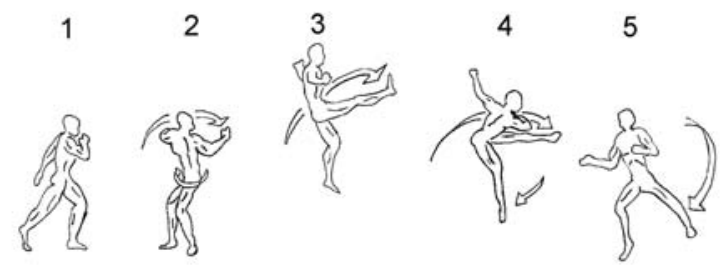

Figura 3 - Fases do Parafuso: (1) Preparação; (2) Rotação de todo corpo; (3) Salto e primeiro chute; (4) Segundo chute da fase aérea; (5) Aterrissagem.
Um outro golpe classificado como parte dos golpes básicos $(4,5)$ é o "Martelo" (Figura 4). Este é um golpe rápido no qual o executante, partindo da "ginga", executa um chute alto no sentido medial. Da mesma forma que os dois últimos golpes apresentados acima, as implicações das forças externas, em particular das componentes da FRS, que agem sobre o sistema são desconhecidas.
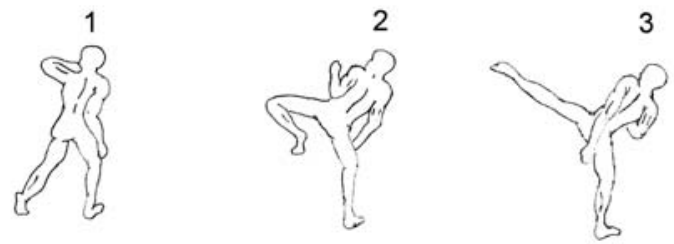

Figura 4 - Fases do Martelo: (1) Preparação com rotação lateral da perna de apoio; (2) e [3] Chute alto com o pé voltado medialmente.

As condições mecânicas de realização dos movimentos descritos sugerem que sua execução pode gerar expressivas cargas externas. Entretanto inexistem dados sobre este tema na literatura especializada.

\section{MATERIAL E MÉTODOS}

\section{Amostra e condições de coleta}

A amostra foi constituída por 10 voluntários, sendo 8 homens e 2 mulheres $(25,1 \pm 3,03$ anos, $172,7 \pm 7,5$ $\mathrm{cm}, 70,35 \pm 7,26 \mathrm{Kg}$ ). Os voluntários eram oriundos de 4 diferentes grupos de capoeira, e o tempo médio de prática da amostra foi de 7,7 $\pm 4,4$ anos.

Os voluntários foram submetidos à Anamnese Ortopédica, através da qual rastreou-se dados relativos a lesões e/ou patologias osteomioarticulares, intervenções cirúrgicas sofridas anteriormente, desvios posturais, bem como outras possíveis alterações de saúde. Nenhuma alteração de saúde capaz de comprometer a prática da Capoeira foi identificada através da Anamnese. Os participantes também assinaram um termo de consentimento autorizando sua participação no estudo e a divulgação dos resultados obtidos. As condições de coleta consistiram de 4 tarefas realizadas, sempre que possível, em duplas de modo a garantir maior validade externa para a execução dos movimentos. As tarefas estudadas consistiam das seguintes seqüências de movimentos: 1 - o voluntá- 
rio avaliado executa a "Negativa Fechada" ao receber o "Martelo" do adversário; 2 - o voluntário avaliado executa a "Armada Pulada"; 3 - o voluntário avaliado executa o "Martelo"; 4 - o voluntário avaliado executa o "Parafuso". Em todas as tarefas, para executar os golpes os voluntários partiam sempre da "ginga" e desferiam o golpe sobre a plataforma de força. Antes do início das coletas os voluntários puderam praticar o movimento sobre a superfície de medição da plataforma, objetivando a redução do efeito retroativo. Para cada uma das seqüências foram coletadas 5 tentativas válidas.

\section{Instrumentos de medição}

As variáveis relacionadas à FRS foram medidas em uma plataforma de força $(600 \times 900 \mathrm{~mm})$ KISTLER AG (9287 A) cujos transdutores de força são do tipo piezoelétricos.

Os sinais coletados por cada um dos quatro conjuntos de três transdutores piezoelétricos são enviados por meio de cabos e interruptores a um amplificador de sinais de oito canais (KISTLER AG, 9865 B). Estes amplificadores de soma e divisão são programados automaticamente para a determinação das três componentes (Fx, Fy e Fz) da FRS, dos momentos (My, Mx e Mz) na superfície da plataforma, das coordenadas (Ax, Az) do centro de pressão, bem como do coeficiente de atrito (11). Os sinais são, então, enviados para um sistema digital, cujo conversor A/D possui 12 bits de resolução e trabalhou numa freqüência de amostragem de $1000 \mathrm{~Hz}$. O gerenciamento deste sistema na coleta, análise e armazenamento dos dados do experimento foi realizado pelo programa de funções BIOWARE (Biomechanical Software Analysis System and Performance, 282A1-20).

\section{Variáveis Analisadas}

Fy(máx): Diz respeito à Força Vertical Máxima alcançada nas aterrissagens dos movimentos 1,2 e 4 e no apoio durante o chute do movimento 3 . Sua intensidade foi normalizada em função do peso corporal (PC) do executante.

$T$ Fy(máx): Relaciona-se com o tempo decorrido entre o instante inicial das aterrissagens dos movimentos 1,2 e 4 e no apoio do chute do movimento 3 , e o instante em que $F y$ (máx) é atingida.
GC Fy: O Gradiente de Crescimento da Força Vertical é o coeficiente que indica a razão entre a $F y(m a ́ x)$ e o $T$ Fy (máx).

I50: É o Impulso gerado por Fy nos primeiros $50 \mathrm{~ms}$ do contato com a plataforma em cada uma das quatro tarefas executadas.

\section{Tratamento estatístico}

Para análise das diferenças entre as médias das variáveis analisadas em cada movimento foi utilizado Teste T de Student Independente processado no software STATISTICA '99 Edition. O nível de significância $(\alpha)$ foi considerado para $p<0,05$.

\section{ANÁLISE E DISCUSSÃO DOS RESULTADOS Análise da sobrecarga no aparelho locomotor Força vertical máxima}

A Tabela 1 apresenta os valores médios e respectivos desvios-padrão (DP) para as variáveis dinâmicas estudadas.

Tabela 1 - Média e desvio-padrão (DP) dos valores de variáveis relacionadas à FRS para os movimentos selecionados da Capoeira.

\begin{tabular}{|c|c|c|c|c|}
\hline & $\begin{array}{c}\text { Fy (máx) } \\
\text { (PC) }\end{array}$ & $\begin{array}{c}\text { TFy (máx) } \\
\text { (ms) }\end{array}$ & $\begin{array}{c}\text { GCFy } \\
{\left[P C \cdot s^{-1}\right]}\end{array}$ & $\begin{array}{c}150 \\
{\left[\cdot 10^{-2}\right.} \\
\mathrm{kg} \cdot \mathrm{ms}]\end{array}$ \\
\hline $\begin{array}{l}\text { Negativa } \\
\text { Fechada }\end{array}$ & $\begin{array}{c}1,91 \\
(0,36)\end{array}$ & $\begin{array}{c}583,00 \\
(290,63)\end{array}$ & $\begin{array}{c}4,91 \\
(4,69) \\
\end{array}$ & $1,53(0,76)$ \\
\hline $\begin{array}{l}\text { Armada } \\
\text { Pulada } \\
\end{array}$ & $\begin{array}{c}4,67 \\
(1,65) \\
\end{array}$ & $\begin{array}{c}57,25 \\
(31,74) \\
\end{array}$ & $\begin{array}{l}115,93 \\
(79,84) \\
\end{array}$ & $7,54(3,04)$ \\
\hline Martelo & $\begin{array}{c}1,36 \\
(0,17)\end{array}$ & $\begin{array}{c}693,32 \\
(322,50)\end{array}$ & $\begin{array}{c}2,61 \\
(1,70)\end{array}$ & $0,84(0,41)$ \\
\hline Parafuso & $\begin{array}{c}5,15 \\
{[2,06]}\end{array}$ & $\begin{array}{l}125,42 \\
{[78,02]}\end{array}$ & $\begin{array}{c}69,17 \\
{[65,68]}\end{array}$ & $6,38(2,88)$ \\
\hline
\end{tabular}

Nota: $F y$ (máx] = Força Vertical Máxima; $T$ Fy $[$ máx] = Tempo para atingir força Vertical Máxima; GC Fy = Gradiente de Crescimento da Força Vertical; $150=$ Impulso gerado por Fy nos primeiros $50 \mathrm{~ms}$ do contato com a plataforma.

Os menores valores obtidos para a Fy (max) foram obtidos quando da realização do "Martelo"

$(1,36 \pm 0,17 \mathrm{PC})$ e da "Negativa Fechada" $(1,91 \pm 0,36$ $\mathrm{PC})$, enquanto os maiores valores foram observados no "Parafuso" (5,15 $\pm 2,06$ PC) e na "Armada Pulada" $(4,67 \pm 1,65$ PC).

Os valores obtidos para o "Martelo" e para a

"Negativa Fechada" foram inferiores aos descritos na literatura (1) para a corrida. Mesmo envolvendo 
movimentos de salto, o "parafuso" e a "armada pulada" apresentaram menores valores para Fy(máx) do que os descritos na literatura para os saltos realizados no atletismo (1) e nas modalidades coletivas (9). Deve-se ainda destacar que os valores descritos para o "Parafuso" foram superiores aos descritos em um estudo anterior (8). Neste estudo os autores obtiveram valores médios para Fy(máx) da ordem de $2,0 \pm 0,2$ PC. As diferenças encontradas pelo presente trabalho, podem ser explicadas pelo maior número de sujeitos da amostra e, conseqüentemente, maior variabilidade no nível de habilidade dos voluntários estudados.

Para o T Fy (max) os maiores valores foram observados na "Negativa Fechada" (583,00 $\pm 290,63 \mathrm{~ms})$ e no "Martelo" (693,32 $\pm 322,50 \mathrm{~ms})$, e os menores na "Armada Pulada" (57,25 $\pm 31,74 \mathrm{~ms})$ e no "Parafuso" $(125,42 \pm 78,02 \mathrm{~ms})$. Considerando que a duração da fase passiva do movimento, fase que corresponde ao intervalo de tempo no qual não é possível modular a ação muscular em função do estímulo, oscila entre 60 e $100 \mathrm{~ms}$ (1), observa-se que, embora o

"Parafuso" tenha apresentado o maior valor médio para Fy(máx), o tempo para atingir este pico é maior que $100 \mathrm{~ms}$. Desse modo, pode-se considerar que o estímulo imposto pela FRS na aterrissagem desta habilidade proporcionou tempo suficiente para condução de uma resposta do sistema nervoso de maneira a impedir que a força produzida neste intervalo de tempo incidisse apenas sobre as estruturas passivas do aparelho locomotor, em especial ossos e articulações. Este fato aconteceu também na "Negativa Fechada" e no "Martelo". A exceção, todavia, foi a "Armada Pulada", que embora tenha apresentado um menor valor médio para Fy(máx) do que o obtido no "Parafuso", mostrou um tempo para atingir este pico inferior a $60 \mathrm{~ms}$. Pode-se dizer, portanto, também com base nestes dados, que, neste movimento, as cargas incidiram sobre estruturas passivas do aparelho locomotor, diminuindo a segurança dos indivíduos que executaram tal habilidade. Refletindo o comportamento da Fy(max) e do T Fy (max), os menores valores para o GC Fy e para o I50 foram obtidos na "Negativa Fechada" (4,91 $\pm 4,69$ $\left.\mathrm{PC} \cdot \mathrm{s}^{-1} ; 1,53 \pm 0,76 \cdot 10^{-2} \mathrm{~kg} \cdot \mathrm{ms}\right)$ e no "Martelo" $\left(2,61 \pm 1,70 \mathrm{PC} \cdot \mathrm{s}^{-1} ; 0,84 \pm 0,41 \cdot 10^{-2} \mathrm{~kg} \cdot \mathrm{ms}\right)$, enquanto a "Armada Pulada" (115,93 $\pm 79,84 \mathrm{PC} \cdot \mathrm{s}^{-1} ;$ 7,54 $\left.\pm 3,04 \cdot 10^{-2} \mathrm{~kg} \cdot \mathrm{ms}\right)$ e o "Parafuso" $(69,17 \pm 65,68 \mathrm{PC} \cdot \mathrm{s}$ $\left.{ }^{1} ; 6,38 \pm 2,88 \cdot 10^{-2} \mathrm{~kg} \cdot \mathrm{ms}\right)$ apresentaram os maiores valores para estas variáveis. Apesar da diferença entre o I50 da "Armada Pulada" e do "Parafuso" não ter sido estatisticamente significativa, ocorreu diferença significativa entre estes movimentos para o GC Fy (Figuras 5 e 6).

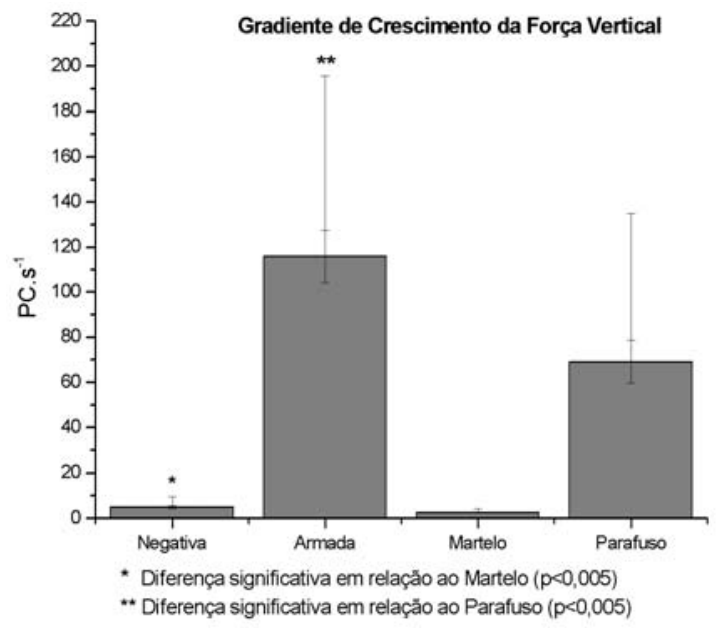

Figura 5 - Média e desvio-padrão do Gradiente de Crescimento de Fy ( $n=50$ ) para os movimentos estudados. (Valores expressos em PC.s-1).

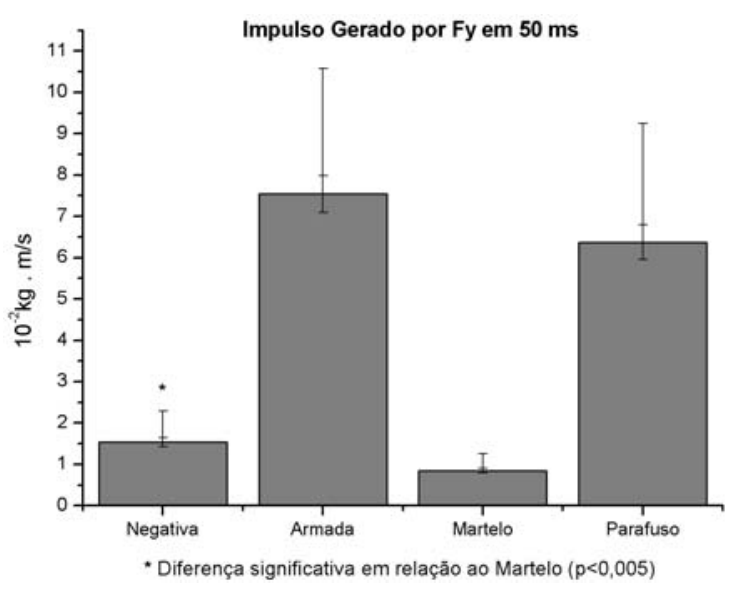

Figura 6 - Média e desvio-padrão para 150 ( $n=50$ ) para os movimentos estudados. (Valores expressos em $\cdot 10-2 \mathrm{~kg} \cdot \mathrm{m} / \mathrm{s}$ ).

Outro aspecto que merece atenção diz respeito a considerável variabilidade observada nos resultados da FRS para os movimentos estudados. Este fenôme- 
no pode ser um reflexo de aspectos como cisões ideológicas de grupos, carência de uma consensual classificação e descrição das habilidades, assim como diferenças individuais no padrão do movimento e nível de habilidade (2). Com isto, os praticantes recebem diferentes orientações pedagógicas nos diferentes grupos de Capoeira, assim como, uma liberdade individual para execução e exploração dos movimentos dentro de um mesmo grupo.

\section{LIMITAÇÕES METODOLÓGICAS DO PROCEDIMENTO EXPERIMENTAL}

O efeito retroativo, manifestado por intermédio da interferência na realização dos movimentos, talvez represente a principal limitação metodológica do estudo. Isto ocorreu devido à intensa preocupação, no ambiente de laboratório, em concretizar a realização dos movimentos dentro dos limites da superfície de medição da plataforma.

Embora os voluntários tenham tido oportunidade de vivenciar as condições de coleta diversas vezes antes que ela fosse efetivamente realizada, isto não apresentou uma solução definitiva para o problema. Tal quadro ficou claro principalmente na realização da "Armada Pulada" e do "Parafuso", movimentos estes que não puderam ser realizados em dupla, em função da reduzida dimensão da plataforma. Além disso, em todas as tarefas houve limitações em função da preocupação dos voluntários em adequar a amplitude de seus movimentos aos limites impostos pela dimensão da plataforma de força.

O movimento mais afetado por esta condição foi a "Negativa Fechada". Esta tarefa foi realizada de forma sincronizada por duplas de voluntários, fazendo com estes conhecessem previamente o momento em que um golpe seria aplicado, situação que difere da condição real de jogo, onde a tomada de decisão dos movimentos a serem executados depende dos movimentos realizados pelo adversário.

\section{CONCLUSÕES FINAIS}

A "Armada Pulada" e o "Parafuso" figuraram como os movimentos que geraram as maiores cargas externas dentre os movimentos da Capoeira estudados. Tomando-se como referência outras expressões do movimento humano (1), pode-se classificar as cargas externas geradas nestes movimentos como sendo de moderada intensidade. Considerando ainda que a carga externa, quando aplicada em intensidade e/ou volume excessivos, pode provocar lesões em diversas estruturas do tecido biológico (10), deve-se dedicar especial atenção à seleção dos movimentos utilizados nas aulas de Capoeira. Ainda que em situações de jogo dificilmente se execute diversas vezes o mesmo movimento, em situações de treinamento, a repetição sistemática de um mesmo movimento é usualmente realizada, aumentando, portanto, as chances de lesões por sobrecarga em indivíduos não preparados e/ou fatigados.

O presente estudo traz evidências de que, pelo menos no âmbito das forças externas, a "Negativa Fechada" e o "Martelo" podem ser inseridos nas fases iniciais do programa de treinamento, ao passo que o "Parafuso" e a "Armada Pulada", movimentos cujas exigências mecânicas e motoras são maiores, poderiam ser inseridos posteriormente no mesmo programa. Do mesmo modo, é imprescindível, em estudos adicionais, relacionar estes aspectos com as diferentes condições, tanto físicas quanto motoras, dos praticantes, bem como as relações entre fadiga muscular e o aumento das cargas externas (12).

\section{CORRESPONDÊNCIA}

\section{Allan Brennecke}

Universidade de São Paulo

Escola de Educação Física e Esporte

Laboratório de Biomecânica

Av. Prof. Mello Moraes, 65

05508-900 - São Paulo - SP - BRASIL

allanbrennecke@yahoo.com.br 


\section{REFERÊNCIAS BIBLIOGRÁFICAS}

1. Amadio AC, Duarte M (Coord.) (1996). Fundamentos Biomecânicos para a análise do movimento humano. São Paulo: Laboratório de Biomecânica da EEFE-USP.

2. Areias A. (1999). O que é Capoeira. 4.ed. São Paulo: Ed. da Tribo.

3. Brennecke A, Matsumoto J, Machado R, Gama W, Serrão JC (1998). Análise bidimensional do deslocamento do centro de gravidade no "bico de papagaio" da capoeira. In: Anais do V Congresso de Iniciação Científica e III Simpósio de Pós-Graduação. São Paulo: EEFEUSP, 106-107.

4. Capoeira N (1992). Capoeira: os fundamentos da malícia. Rio de Janeiro: Record.

5. Capoeira N (1992). Capoeira: pequeno manual do jogador. 5.ed. Rio de Janeiro: Record.

6. Hamill J, Knutzen KM (1999). Cinética Linear. In: Bases Biomecânicas do Movimento Humano. São Paulo: Manole, 394-427.

7. Hayflick L (1996). O envelhecimento da cabeça aos pés. In: Como e porque envelhecemos. Rio de Janeiro: Campus, 157-75.

8. Heine V, Almeida G, Serrão JC (2000). Análise dinâmica do movimento "parafuso" da capoeira. In: Anais do VII Congresso de Iniciação Científica e V Simpósio de Pós-Graduação São Paulo. São Paulo, EEFEUSP, 93-94.

9. McClay JS, Robson JR, Andriacchi TP, Frederick EC, Gross T, Martin P, Valiant G, Williams KR, Cavanagh PR (1994). A profile of Ground Reaction Forces in Professional Basketball. J Appl Biomech 10: 223-236.

10. Radin EL, Orr RB, Kelman JL, Paul IL, Rose RM (1982). Effect of prolonged walking on concrete on the knees of sheep. J. Biomechanics 15: 487-492.

11. Serrão, JC (1999). Aspectos Biomecânicos da Influência do Calçado Esportivo na Locomoção Humana. $164 \mathrm{f}$. Tese Doutorado. Escola de Educação Física da Universidade de São Paulo, São Paulo.

12. Serrão, JC (1996). Estudo do Comportamento Dinâmico da Força de Reação do Solo e Resposta Eletromiográfica em Corredores Induzidos à Fadiga. $123 \mathrm{f}$. Dissertação de Mestrado. Instituto de Biociências Universidade Estadual Paulista, Rio Claro.

13. Silva, GO (1998). Capoeira: do Engenho à Universidade. São Paulo: Coordenadoria de Comunicação Social da Universidade de São Paulo. 